\title{
On the modified random walk algorithm for Monte-Carlo radiation transfer (Corrigendum)
}

\author{
T. P. Robitaille \\ Spitzer Postdoctoral Fellow, Harvard-Smithsonian Center for Astrophysics, 60 Garden Street, Cambridge, MA, 02138, USA \\ e-mail: trobitaille@cfa.harvard.edu
}

A\&A 520, A70 (2010), DOI: 10.1051/0004-6361/201015025

Key words. radiative transfer - diffusion - circumstellar matter - methods: numerical - errata, addenda

In Sect. 2.3, the diffusion coefficient for the modified random walk is given in Eq. (18) as

$D=\frac{1}{3 \rho} \frac{\int_{0}^{\infty} \frac{B_{v}(T)}{\chi_{v}} \mathrm{~d} v}{\int_{0}^{\infty} B_{v}(T) \mathrm{d} v}$.

This equation is correct, but the ratio of the integrals was referred to as "the inverse of the Rosseland mean mass extinction coefficient". This is incorrect, because the Rosseland mean opacity includes $\mathrm{d} B_{v}(T) / \mathrm{d} T$ terms rather than $B_{v}(T)$. Instead, the ratio of the integrals is the inverse of the Planck reciprocal mean mass extinction coefficient (in line with the terminology used by Fleck \& Canfield 1984). We can write this mean mass extinction coefficient as:

$\bar{\chi}_{P^{-1}} \equiv \frac{\int_{0}^{\infty} B_{v}(T) \mathrm{d} v}{\int_{0}^{\infty} \frac{B_{v}(T)}{\chi_{v}} \mathrm{~d} v}$ and the diffusion coefficient for the modified random walk can then be written as:

$D=\frac{1}{3 \rho \bar{\chi}_{P^{-1}}}$.

The reason why one does not recover the Rosseland mean opacity in the case of photon diffusion in the modified random walk is that this random walk occurs in an isothermal medium, for which the derivation of the traditional diffusion coefficient breaks down (since it assumes that temperature gradients are present).

I am grateful to Ant Whitworth for bringing this mistake to my attention, and to Michiel Min for useful discussions.

\section{References}

Fleck, Jr., J. A., \& Canfield, E. H. 1984, J. Comput. Phys., 54, 508 